\title{
Unanticipated bleeding with the etonogestrel implant: advice and therapeutic interventions
}

\author{
Jane Dickson, ${ }^{1}$ Lesley Hoggart, ${ }^{2}$ Victoria Louise Newton ${ }^{3}$
}

${ }^{1}$ Consultant in Sexual and Reproductive Healthcare, Oxleas NHS Foundation Trust, London, UK

${ }^{2}$ Senior Lecturer, The Open University, Milton Keynes, UK ${ }^{3}$ Research Fellow, Faculty of Health and Social Care, The Open University, Milton Keynes, UK

\section{Correspondence to} Dr Jane Dickson, Oxleas NHS Foundation Trust, Memorial Hospital Outpatients Department, Shooters Hill, Woolwich, London SE18 3RG UK; jane.dickson@nhs.net

Received 28 October 2013 Revised 14 April 2014 Accepted 13 May 2014

\section{CrossMark}

To cite: Dickson J, Hoggart L, Newton VL. J Fam Plann Reprod Health Care 2014:40:158-160.

\section{THE PROBLEM}

The etonogestrel contraceptive implant $\left(\right.$ Implanon ${ }^{\circledR}$, Nexplanon $^{\circledR}$ ) is widely recognised as a reliable and cost-effective form of contraception. ${ }^{1}$ However, continuation rates are of concern as the method loses cost effectiveness when discontinued early. It has been calculated that $60 \%$ of implant removals are for irregular or unpredictable bleeding. ${ }^{2}$

Guidelines for standard practice in implant provision emphasise the importance of adequate counselling about side effects, especially bleeding irregularities, before implant insertion. ${ }^{3}$

In our recently published study ${ }^{5}$ we showed that even in cases where young women have been made aware of the possibility of irregular bleeding, the clinician's messages are not always fully absorbed. In this study the women each had their own interpretations of the advice they had been given, and individualised responses to their experience of side effects. It was confirmed that the predominant reason for requests for implant removal was irregular bleeding. It was also evident that:

1 The women had not been prepared for the reality of prolonged bleeding or irregular bleeding patterns, even when they could recall being informed about these at their initial consultation.

2 They often persevered with their implants, waiting for the bleeding pattern 'to settle', so often tolerated significant amounts of discomfort and inconvenience.

3 Bleeding in conjunction with other side effects was particularly problematic.

Patients are often advised to 'persevere' with the method in the anticipation that their symptoms may ultimately resolve. However, if the situation does not improve, the 'therapeutic window' for some form of intervention is missed. In our study, the women had reached a 'tipping point' at which intervention was no longer possible. Our study showed the importance of recognising the real impact of troublesome bleeding: the impact it has on sex life, relationships and finances, and the anxiety caused by unpredictability. It is important to intervene as soon as possible when bleeding is presented as a problem, rather than encouraging acceptance of the situation.

Research into methods of controlling the bleeding problems related to contraceptive implants is inconclusive, so a pragmatic approach to management should be employed. ${ }^{6}$

\section{BACKGROUND TO BLEEDING}

Continuous low-dose progestogen, as released by the implant, predisposes to breakthrough bleeding because uterine blood vessels proliferate and become disordered, with a 'leaky' basement membrane. There is decreased glandular and stromal support and reduced epithelial integrity. ${ }^{7}$ At the opposite extreme, highdose progestogen leads to pseudodecidualisation and endometrial stability. Estrogen stimulates proliferation of the endometrial stroma which is also beneficial in supporting the endometrium.

Prostaglandins are also important in the mechanisms that control uterine bleeding. Non-steroidal anti-inflammatory drugs (NSAIDs) (e.g. mefenamic acid) therefore have a potentially effective role in the management of troublesome bleeding through inhibition of inflammatory prostaglandin production.

\section{MANAGEMENT OPTIONS}

Guidance for the control of unscheduled bleeding related to hormonal contraception $^{8}$ recommends that other possible causes for bleeding are initially excluded (e.g. exclusion of sexually transmitted 
infection, pregnancy and other genital tract pathology). Once the clinician is confident that the cause of the bleeding is implant-related a number of options are available.

1 The best approach is to provide estrogen, usually in the form of the combined oral contraceptive pill (COC). This can be given 'off-licence' either cyclically or continuously, initially for 3 months. If symptoms resolve, but then deteriorate when treatment is stopped, a COC can be given for the entire duration of implant use if necessary. FSRH guidance ${ }^{3}$ states that use for longer than 3 months is a matter of clinical judgement. One of the preparations containing ethinylestradiol $30 \mu \mathrm{g}$ and levonorgestrel $150 \mu \mathrm{g}$ is ideal, as this is a relatively progestogenic combination. There is no reason why this regime cannot be commenced as soon as abnormal bleeding becomes a problem. There is evidence to support the use of estrogen in the management of bleeding with other progestogenic methods, ${ }^{9}$ and it is this evidence that was extrapolated to provide the FSRH guidance.

2 In cases where estrogen use is contraindicated (e.g. focal migraine), a desogestrel progestogen-only pill (POP) has sometimes been advocated to try to alleviate bleeding. However, particularly in the early stages of implant use, this can compound the problem by contributing to the 'low-dose progestogen' instability. In this situation, a better intervention would be to use a NSAID such as mefenamic acid. There is no indication to use an antifibrinolytic agent (e.g. tranexamic acid) as the mechanism of these drugs is to impair plasmin activation. This action is not important in the management of troublesome implant bleeding, unless there is associated menorrhagia. The desogestrel POP may have a role in helping bleeding where implant bleeding has been stable but subsequently has become troublesome (e.g. after 1 or 2 years of use).

3 There has been research to study the effect of the antibiotic doxycycline on the troublesome bleeding caused by the implant. It had been proposed that as a powerful inhibitor of matrix metalloproteinases, enzymes responsible for endometrial breakdown and remodelling, it may be beneficial. However, one randomised controlled trial ${ }^{10}$ concluded that the benefits of its use are not significant. Nevertheless, this does not mean that it may not have a beneficial effect if there is co-existent chlamydial infection or the possibility of Mycoplasma genitalium that may be contributing to endometrial instability.

4 Finally, there is a role for high-dose progestogens when short-term arrest of bleeding is required (e.g. norethisterone $5 \mathrm{mg}$ tds).

\section{PATIENT COUNSELLING}

In order to maximise compliance with the contraceptive implant, clinicians need to demonstrate an empathetic and proactive approach to the management of bleeding problems. From the time that contraceptive counselling is first undertaken, bleeding patterns should be discussed with patients, including advice about an 'open-door approach' to the management of such problems. It needs to be acknowledged that as soon as the bleeding pattern is distressing to the patient, it is a problem.

There are no guarantees that perseverance with an implant without intervention will ensure improvement or acceptance of the bleeding, but we have seen that a 'wait and see' approach to bleeding has often been employed. In fact, if a woman is encouraged to retain the implant and there is no resolution, she may lose faith in her medical professional. ${ }^{45}$ The FSRH guidance on implants ${ }^{3}$ states that "women using progestogen-only implants should be advised that no routine follow-up is required but that they can return at any time to discuss problems or change their contraceptive method". We would suggest that women are actively encouraged to return as soon as bleeding is problematic as early intervention may lead to improved implant retention.

If there is no contraindication, the COC is the most effective intervention. If there are contraindications to the COC, mefenamic acid (e.g. $500 \mathrm{mg}$ tds for 1 week) can be given. Other management options may be tried on a pragmatic but non-evidence-based basis.

Competing interests None.

Ethics approval Pan London Ethics Committee from Brent Research Ethics Committee.

Provenance and peer review Not commissioned; externally peer reviewed.

\section{REFERENCES}

1 Mavranezouli I. The cost effectiveness of long-acting reversible contraceptive methods in the UK: analysis based on the decision-analytic model developed for the National Institute for Health and Clinical Excellence (NICE) clinical practice guideline. Hum Reprod 2008;23:1338-1345.

2 Rai K, Gupta S, Cotter S. Experience with Implanon in a north east London family planning clinic. Eur J Contracept Reprod Health Care 2004;9:39-46.

3 Faculty of Sexual \& Reproductive Healthcare Clinical Effectiveness Unit. Progestogen-Only Implants. February 2014. http://www.fsrh.org/pdfs/CEUGuidanceProgestogenOnly Implants.pdf [accessed 13 April 2014].

4 Hoggart L, Newton VL. The experience of side effects from contraceptive implants: a challenge to bodily control among young women. Reprod Health Matters 2013;41:196-204.

5 Hoggart L, Newton VL, Dickson J. 'I think it depends on the body, with mine it didn't work': explaining young women's decisions to request subdermal contraceptive implant removal. Contraception 2013;88:636-640.

6 Mansour D, Bahamondes L, Critchley H, et al. The management of unacceptable bleeding patterns in etonogestrel-releasing contraceptive implant users. Contraception 2011;83:202-210.

7 Livingstone M, Fraser IS. Mechanisms of abnormal uterine bleeding. Hum Reprod Update 2002;8:60-67. 
8 Faculty of Sexual \& Reproductive Healthcare Clinical Effectiveness Unit. Management of Unscheduled Bleeding in Women Using Hormonal Contraception. May 2009. http://www.fsrh.org/pdfs/ UnscheduledBleedingMay09.pdf [accessed 21 October 2013].

9 Abdel-Aleem H, d'Arcangues C, Vogelsong K, et al. Treatment of vaginal bleeding irregularities induced by progestin only contraceptives. Cochrane Database Syst Rev 2007;4: CD003449.

10 Weisberg E, Hickey M, Palmer D, et al. A randomised controlled trial of treatment options for troublesome uterine bleeding in Implanon users. Hum Reprod 2009;24:1852-1861. 